\title{
A Survey on the Flexibility Requirements Related to Business Processes and Modeling Artifacts
}

\author{
Selmin Nurcan ${ }^{1,2}$ \\ ${ }^{1}$ Université Paris 1 Panthéon Sorbonne, Centre de Recherche en Informatique; 90, rue de Tolbiac 75634 Paris cedex 13 France \\ ${ }^{2}$ IAE de Paris Sorbonne Graduate Business School; 21, rue Broca 75005 Paris France \\ Selmin.Nurcan@univ-paris1.fr
}

\begin{abstract}
In competitive and evolving environments only organizations which can manage complexity and can respond to rapid change in an informed manner can gain a competitive advantage During the early 90's, workflow technologies offered a transversal integration capacity to the enterprise applications. Today, to "integrate" enterprise applications -and the activities they support- into business processes is not sufficient. The architecture of this integration should also be flexible. Enterprise requirements highlight flexible and adaptive processes whose execution can evolve (i) according to situations that cannot always be prescribed, and/or (ii) according to business changes (organizational, process improvement, strategic ...). More recent works highlight requirements in term of flexible and adaptive workflows, whose execution can evolve according to situations that cannot always be prescribed. This paper presents the state of the art for flexible business process management systems and criteria for comparing them.
\end{abstract}

\section{Introduction}

Over the two last decades, market changes have led to a business environment that is constantly evolving. Companies change to better satisfy customer requirements, improve internal processes and adapt their products and services. At the same time, they also experienced the effects of the integration and evolution of information and communication technologies. Thus, IT and management go hand by hand in the way of reacting, adapting and implanting new ways of doing business. Companies need (i) to integrate their new solutions with their legacy systems in a global IT architecture and (ii) to orchestrate the execution of their activities and the use of the supporting technological solutions in an integrated environment. The capacity of quick reaction of organizations is mainly due to the ability of handling the support systems in favour of the business evolution requirements.

The production of added value in business value chains is highly dependent of the quality of their supporting information systems and the effective strategic use of information technologies. In all management challenges, information systems (IS) should be continuously adapted to changing business practices and needs. As a consequence, information systems are becoming more and more complex and shall be conceived in the wider strategic context of organizations management. This can be achieved by developing process-centric solutions. The paradigm of Business Process Management stresses the importance of integrating entire processes rather than simply integrating data or applications. The aim is to design and control the organizational structures in a very flexible way so they can rapidly adapt to changing environments. During the early 90's, Workflow Management Systems (WFMS) have been positioned as appropriate technological solutions for integrating process islands at a high level so that they can collaboratively provide business solutions that each individual application is unable to provide. However, the formalisms developed for the specification of workflow definitions were -and still largely are- almost systematically activity oriented. Consequently, process definitions have the advantage to be easily transformable in executable code but the disadvantage of being prescriptive and rigid.

Flexibility has been the focus of many researches [42], [44], [50], [52], [56]. There are many definitions of the flexibility in literature [54]. It is defined in [42] as "the ability to yield to change without disappearing". We consider the flexibility as the capacity of making a compromise between, first, satisfying, rapidly and easily, the business requirements in terms of adaptability when organizational, functional and/or operational changes occur; and, second, keeping effectiveness. Processes flexibility means fast reactivity to internal and external changes. It reflects the easiness to make evolve business process schemes (when required). Flexibility is also reflected by the ability that the support systems have to take into account business changes.

The objective of the research in progress is to measure the capacity of the modeling solutions provided in the literature to represent the flexibility requirements of current business processes being intra or inter organizations.

This paper is organized as follows: Section 2 presents a 
survey on business process modeling, regarding modeling perspectives, modeling formalisms and the commercial offer. Section 3 proposes a state of the art for flexible process modeling and controlling.

\section{Business process modeling: a survey}

Business processes (BPs) can be roughly classified into two categories depending on their nature. The first concerns well-defined and -often- repetitive processes having important coordination and automation needs. The second category concerns ill-defined processes. The essential preoccupation with the latter is the information and knowledge sharing between the actors implied in the processes more than the coordination of their tasks. Business processes of this category require evidently more flexibility. For many organizations, well-defined and illdefined processes coexist and must be handled in the final business model [40].

\subsection{A classification of business process modeling perspectives}

Literature suggests that existing approaches to enterprise knowledge modeling can be classified into two categories. In the first category, an organization is represented as a set of inter-related elements satisfying common objectives. For instance, VSM [17] allows us to model an organization as a set of viable sub-systems representing respectively the operation, co-ordination, control, intelligence and politics aspects of an organization. The second category, Enterprise modeling, refers to a collection of conceptual modeling techniques for describing different facets of the organizational domain including operational (information systems), organizational (business processes, actors, roles, flow of information etc), and teleological (purposes) considerations [5], [13], [24], [39], [64]. Commercial Workflow Models belong also to this category.

Business process (BP) modeling usually combines three basic views: (i) the functional view is expressed based on Data Flow Diagrams [32]; (ii) the behavioral view focuses on when and under which conditions activities are performed; this view is described using state diagrams or interaction diagrams [22]; and (iii) the structural view focuses on static aspects of processes capturing the manipulated objects [46].

Five perspectives were proposed in [58] and extended in [12] with a sixth one: the intentional perspective. The functional perspective characterizes the activities that have to be performed during a process. The process perspective characterizes the conditions to execute a process. The organization perspective reflects information about the organizational structure and actors to which the BP is intended. The information perspective covers data handled by BPs. The operation perspective describes elementary operations performed by resources and applications. Last but not least, the intentional perspective represents goals and strategies that the enterprise implements in its processes. A modeling approach does not necessarily offer concepts to cover the six perspectives. For instance, IDEF0 covers the functional perspective and touches lightly on the information perspective [32]; IDEF1 covers the information perspective and IDEF3 completes them with the process one. To represent flexible processes, a modeling solution has to provide a minimum set of perspectives to represent the enterprise elements that are potentially impacted by changes.

\subsection{Modeling formalisms}

Literature provides various process modeling formalisms that we classify into four categories: activity oriented, product oriented, decision oriented and conversation oriented models. Each category has its underlying paradigm that may be examined in terms of its appropriateness to flexible process modeling.

Activity-oriented models allow us to prescribe a process as a set of activities to be performed and their relationships regarding pre-defined control and data flows [22], [32]. Those formalisms are useful for representing the functional view of BPs. Nevertheless, the linear view of activity decomposition is inadequate for modeling illdefined BPs, particularly if the latter suffer frequent changes and/or alternative choices are based on human decisions instead of calculable arguments. Activityoriented models are still dominant in the literature. It is stated in [4] that "in a process centered environment, a process (model) plays the role of a program to be executed in order to control and manage the process (instance)". Many activity-oriented models are based on this hypothesis in despite of the criticism of [29], which argues that process programming only allows one to represent the well assimilated parts of processes and not the creative parts.

Product-oriented models do not put forward the activities of a process but rather the result of these activities. They model the evolution of the product and couple the product state to the activities that generate this state [22]. These models are useful for tracing the transformations performed and their resulting products, i.e. business objects, products or services in our case. They are used for representing the structural and behavioral views (§2.1) and are more appropriate than activity-oriented ones for representing ill-defined BPs. However as far as flexibility is concerned and considering the highly nondeterministic nature of ill-defined processes, it seems difficult to write down a realistic state-transition diagram that adequately describes what has to happen.

The most recent type of process models [14], [18], [38], [39] is based on the decision-oriented paradigm 
according to which the successive transformations of the product are looked upon as consequences of decisions. The underlying philosophy is that a process model does not have only to specify the linking of activities or product states but also the intention behind the execution of activities and their linking. Decision-oriented models are semantically more powerful than the two others because they explain not only how the process proceeds but also why. Their enactment guide the decision making process that shapes the business, help reasoning about the rationale of decisions [33]. This paradigm seems to be the particularly appropriate for representing ill-defined BPs [5] or processes requiring flexibility [38].

Conversation models are based on the speech act theory and on the principle that each sentence expressed by someone represents an intention, a commitment [53]. The Action model [34] which is amongst actioncoordination systems seems more appropriate for modeling ill-structured processes than activity-oriented models. It defines a structure to represent the conversation relationship between two participants, customer and performer. Commitments are contained within these structures which can be divided into four phases of interaction: preparation, agreement, performance and acceptance. This structure can also help to consider optimization possibilities. In fact, highlighting the organizational reasons which cause the communications between some roles can justify changes of processes.

Each of those formalisms uses one or several perspectives presented in §2.1. For instance, STATEMATE [22] deals with the traditional "who, what, where, when and how" perspectives using activity, state and module charts while IDEF0 [45] employs a data flow perspective to model processes. Activity and product-oriented models have similar expressivenesses. However this is not sufficient for modeling processes where human reasoning is a major component. Decision-oriented models seem more appropriate to highlight why decisions are made and to facilitate the introduction of change.

More recent BP modeling languages still provide concepts for activity-oriented and product-oriented representations. The Unified Modeling Language (UML) allows not only modeling application structures, behaviors and architectures, but also BPs. Event-Driven Process Chains (EPC) represent temporal and logical dependencies between functions, events or connectors which are linked via control flows. An interchange format for EPCs, EPC Markup Language (EPML), has been proposed in [35]. The Business Process Management Initiative (BPMI) developed open specifications to enable the standardsbased management of cross-enterprise processes based on BPM Systems. The Business Process Modeling Language (BPML) defines a formal model for expressing abstract and executable processes that address all aspects of BPs, including activities of varying complexity, transactions and their compensation and exception handling. The Business Process Modeling Notation (BPMN) defines a BP model as a network of activities and control flows. It is presented as a notation understandable by all users, from business analysts to developers and business actors.

A conceptual modeling framework offering the rigor necessary for modeling well-defined BPs, and the flexibility and adaptability required for ill-defined BPs was proposed in [37]. This framework gives the ability to describe the invariants of the organization in terms of objectives and strategies before specifying the manner of making them operational in a particular organizational situation. The vision of the organization which is promoted is structured according to three layers of concern. The objectives of the organization are achieved by implementing the enterprise processes whose are themselves supported by the enterprise information systems. The two first layers focus on intentional and organizational aspects of the enterprise, i.e. the business objectives and how these are achieved through the co-operation of enterprise actors manipulating such enterprise objects. The third one focuses on system aspects i.e., application components that will support the enterprise, its processes and its actors in order to achieve the business objectives.

Despite innovative works proposed by the BP community, there is a lack of approaches that support variability according to the contextual requirements of each BP instance. Due to the economic and technological progress, customers' expectations are becoming imprecise and varied following the context in which expectations are formulated. Hence, context related knowledge becomes an essential resource to adapt the behavior of BPs. A conventional BP model may fit customers' needs in a given context and not in another one. The ability to integrate the context related knowledge allows BP models to be active, flexible, fine-grained and able to express a variety of business rules. In [50], a role driven approach for was developed for supporting context awareness in flexible BPs. It has two major benefits: (i) it offers flexibility in assigning functions to roles since a function can be performed by several possible roles according the performance context rather than a specific one, and (ii) it gives to actors some autonomy allowing them to develop strategies for performing operations, operational goals and functions. In [51], challenges related to the development of a new promising paradigm for BP modeling supporting explicit definition of the 'context related knowledge' are identified. Authors argue that any information reflecting changing circunstances during the execution of a BP can be considered as contextual information. The context is thus defined as "the collection of implicit assumptions that is required to activate accurate assignments in the $\mathrm{BP}$ model at the process instance level”. In [51], the role-driven BP modeling approach (RBPM) presented in [50] was extended to support context awareness. 


\subsection{Position of workflow softwares for modeling and enacting processes}

The workflow concerns, at first, an activity of scheduling and coordination of work between actors implicated in a business process. The aim of workflow analysis is to find the right division of a given work process into tasks and an ordering among these tasks leading thus to the representation of a "procedure". Thereafter, WFMSs provide mechanisms to execute the defined flow of tasks [16]. Several classifications have been proposed for workflow applications. The commonly used was defined in [30] and divides workflows into three classes, depending on the nature of the processes they support and the value these processes have for the enterprise. An additional category (collaborative) was mentioned in [2] leading the categorization into four classes.

- Production workflows involve repetitive and predictable BPs. They implement the core processes of the enterprise and incorporate access to various ISs through workflow activities. This is the closest category to the commercial WFMS solutions and to the generic workflow product structure adopted by WfMC [63].

- Administrative workflows involve repetitive, predictable processes with simple task coordination rules and do not concern the core processes of the enterprise. They can be automated using a repositoryoriented WFMS as the production workflows but also message-oriented ones where process definitions can be part of the messages and not stored in a repository.

- Collaborative workflows include iterative tasks over the same step until some form of agreement has been made. It seems very difficult to model those using classical WFMSs and the underlying activity-oriented (prescriptive) models since it is impossible to predefine the steps to follow. Most of the co-ordination is done by human participants.

- Ad hoc workflows have no predefined structure. Workflow support is limited to the provision of communication mechanisms to route case (process instance) data between workers and possibly some support for logging and state tracking. Ad hoc workflows tend to be created to deal with exceptions. The coordination is controlled by human participants.

A great extend of the process models offered by the commercial solutions are activity-oriented and are devoted to the representation of BPs whose execution could be automatically supported by a WFMS based on the same paradigm. The Workflow Management Coalition defined the workflow as 'The automation of a business process, in whole or part, during which documents, information or tasks are passed from one participant to another for action, according to a set of procedural rules' [63]. The modeling formalism adopted by the WfMC is also activity oriented.
In terms of automated support for executing BP models, commercial WFMS and the underlying control flow models are useful for well-defined and repetitive work processes (production and administrative). Nevertheless, they cannot be used for ill-defined BPs (ad hoc and collaborative) neither to deal with the dynamic modification of well-defined ones. Recent works underline the needs in term of flexible and adaptive workflows [9], [58,] [62] whose execution can evolve according to situations that cannot always be prescribed.

Few WFMSs (InConcert, Ensemble and TeamWARE flow) allow creating and modifying process definitions during their execution. For instance, Inconcert supports the definition of process models by discovering, i.e. by induction from process instances [59]. [10] deals with the issue of the coordination of cooperative activities and presents a set of requirements -including flexibility and dynamicity- for a WFMS that aims to support cooperative workflow. In [49] a classical workflow model is combined with some pocket of flexibility that reduces the constraints on execution. In [6], the artifact of emergent workflow is proposed to support the adaptation of process instances at runtime. In [61], the authors suggest a set of changes patterns and change support features to foster systematic comparison of existing process management technology with respect to change support. Based on these change patterns and features, they provide an analysis and evaluation of the studied systems.

\subsection{Discussion}

Since the introduction of the process centered view of organization management by M. Hammer and J. Champy, $\mathrm{BP}$ modeling gained importance in both the management community and the system engineering community. Through a better understanding obtained by an explicit representation of their processes, the former aims to improve organization performance via process reengineering whereas the latter aims to design/redesign the IT solution that best fit the reengineered processes. In order to respond to this demand, a large number of process models were developed as well as tools to support their definition and management over time. Most of these models concentrate on Who does What, When, i.e. on the description of the operational performance of tasks to produce results. Modeling BPs consists in capturing processes and highlighting significant aspects of the business. During the two late decades, several languages dealing with BP modeling were proposed: traditional input-process-output languages, conversation-based languages, languages based on role modeling, system thinking and system dynamics techniques, and constraintbased languages.

The literature shows a convergence on a set of concepts such as procedure, task, role, actor, resource, 
decomposition of tasks... and highlights the fact that an appropriate model for representing BPs should also provide means to represent ill-structured ones.

The concept of role is common to the studied models. A role is the definition of an organizational intention shared by a collection of users, all of whom have the same privileges and obligations to a set of work processes in an organization. It seems to be the main concept for the representation of BPs. Nevertheless approaches dealing with role descriptions are not satisfactory to meet flexibility requirements. For instance, Role-InteractionNetworks [55] and Role-Activity-Diagrams [41], represent roles as sets of ordered activities or interactions. They introduce "swim-lines" to indicate responsibilities of participants. They also describe interactions between couples of roles, from a source to a target role. In addition, [3] improves the readability of BP models by making roles explicitly present. Its main contribution with respect to [41] and [55] is to represent explicitly physical objects that a role needs to execute its actions. Nevertheless, it does not allow this sequence of actions to be performed by actors having different competencies, according to the situations in hand.

Among modeling techniques found in the literature, those based on role modeling have the advantage of supporting the well-known separation of duties principle (SoD). "The purpose of the SoD is a policy to ensure that failures of omission or commission within an organization are caused only by collusion among individuals and, therefore, are riskier and less likely, and that chances of collusion are minimized by assigning individuals of different skills or divergent interests to separate tasks" [19]. Furthermore, the concept of role not only allows underlining the responsibility of each actor and reflects the organizational structure but also improves the understanding of the way responsibilities are achieved. Adopting role based methods to represent BPs is useful, particularly if they are flexible enough to meet BP flexibility requirements, especially organizational, functional and operational requirements.

Context-awareness [51] allows expressing a rich set of business rules and to adjust assignment activation and deactivation in a flexible way offering practical alternatives that depend on the context. It provides more appropriate matching so that only an actor which plays the appropriate role can perform an operation and only the suitable functions will be included in a given BP, etc. This ensures that BP instantiation matches actual usage and needs. As well, a great amount of flexibility is brought by the concept of context.

Social and organizational factors take an important place in any organization. A BP model must capture much more than the steps of 'procedures'. The concept of goal expresses an intention. Goals are high level objectives of the organization. The more recent goal-oriented approaches [36], [37] define stable characteristics of the business that any organization choice must respect. Compound goals can be decomposed into sub-goals. At the most detailed level, the way of achieving the operationalizable or atomic goals should be specified in terms of actors and activities, i.e. operational concerns. We argue that knowledge intensive business processes require modeling artifacts which can represent the organizational goals, strategies, responsibilities and risks rather than exclusively actors, operations and activities.

\section{Flexible process modeling and controlling : State of the Art}

According to [63], a process definition consists of a network of activities and their relationships, criteria to indicate the start and termination of the process, and information about the individual activities such as participants, associated IT applications supporting them and data, etc. A process instance represents a single enactment of a process definition. The process definition adopted by WfMC corresponds to a prescriptive process model in the sense that "how things must/should/could be done" should be pre-defined before the enactment of the process definition. Remind that, in opposite, a descriptive process model aims at recording and providing a trace of what happens during the business process [20].

We studied several flexible/adaptive workflow approaches of the literature. Figure 1 shows the properties of these approaches we will discuss below and situates them in the workflow applications life cycle. Figure 2, at the end of this section, will summarize the properties we identified and analyzed with respect to business process flexibility requirements in a global picture. These properties are developed underneath.

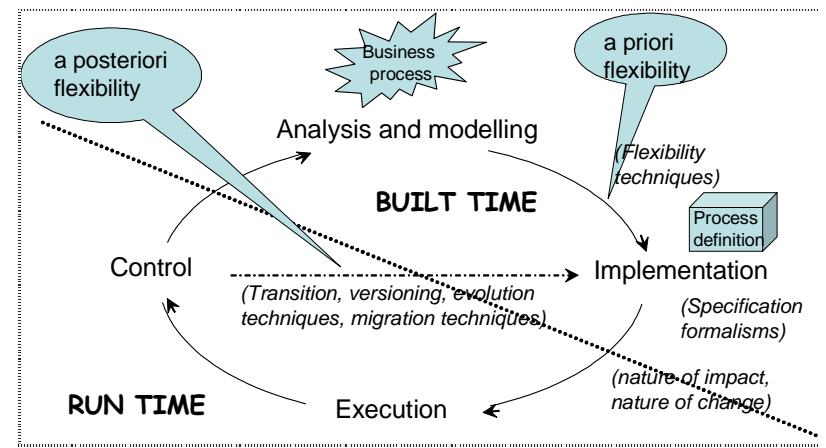

Figure 1: Workflow applications life cycle and properties about flexibility

The nature of the flexibility drew first our attention. This defines if the capacity of taking into account the environmental change should be incorporated in the process model during the build-time or not.

The flexibility by adaptation (a posteriori) allows 
adapting the process definition or its instances during their execution. This is the most usual case in the literature. The principal challenge is to know (i) when and according to which criteria the adaptation should be done? (ii) in the type or the instance level? (iii) ? how to deal with the instances which are currently running? Approaches which offer only this kind of flexibility are based on prescriptive modeling formalisms. It could be considered that the resulting process definitions are not really flexible but rather adaptive. In fact, these approaches can not anticipate the capacity to change during the build-time. Prescriptive modeling formalisms are well adapted to specify BPs which require high degree of control and prediction and for which the need for change remains an exception. This concerns production workflows. We consider that a process definition which is often candidate to transformations suffer from the weakness of the underlying modeling formalism; the latter is too rigid to be able to capture the dynamic nature of the BP.

The flexibility by selection (a priori) is based on modeling formalisms which can offer the capacity to deal with the environmental change without any evolution of process definitions [8], [8], [11], [23], [26], [28], [47], [57], [60], [62]. This means that this capacity should be incorporated in process definitions during build-time. The process definition should be specified in a sufficiently flexible way so that it will yield under the influence of the environment without breaking. Thus process instances are always in conformity with the process definition. Accordingly, the workflow enactment service should be able to execute 'incomplete' specifications of process definitions. Thus, it depends on the user decisions for the selection of (i) a process component (dynamic construction of the process instance) [31], (ii) an execution path among several possible ones, (iii) a behavior to associate to a process component (actor, activity, resource, ..) , (iv) a wayof-doing to perform an activity [18] or (v) the strategy to adopt in the interpretation of the process model by the enactment engine [7].

One of the studied approaches adopts only the a priori flexibility [31]. It is based on the dynamic construction of instances by selection of components in a library. The COO-flow project [21] is somewhat different from the other approaches adopting the a priori or the a posteriori flexibility. It, however, shares a similarity with the AFLOWS approach [7]. In both cases the flexibility is introduced (a priori) into the enactment engine. COO-flow introduces the flexibility in the control flow by anticipation and in the data flow by the transmission of intermediate results, i.e. by communication. It is recommended, for approaches using the a priori flexibility, to allow adapting a process instance when the system can not deal with a not anticipated event using its a priori flexibility capacities [1], [7], [14], [18], [21], [27], [38].

Nature of impact defines if the change will impact the process definition or instances. It is only applicable when the flexibility is a posteriori.

Local: The transformation concerns instances. It is necessary to detect if it can have indirect impacts on other instances [14], [18], [26], [27]. For instance, Adept flex [11] expresses the semantics links existing between process instances.

Global: The transformation concerns the process definition [1], [8], [8], [11], [23], [28], [38], [47], [57], [60], [62]. The classical problem concerns the current instances of this type. Is it possible to propagate the transformation on the current instances?

Nature of change defines why the process transformation is required. It is only applicable when the flexibility is a posteriori.

Ad hoc: the transformation is dynamically performed on one or several instances when the process definition is not convenient for the execution conditions [14], [18], [27], [60]. The ad hoc change has thus a local impact.

Corrective: the transformation aims to correct a design error on the process definition or to react to an exception which happens during the execution of an instance [8], [26], [38]. It can have local or global impacts.

Evolutionary: the transformation is required due to the redesign or reconfiguration of the BP [28], [47], [57], [60]. The old process definition is than considered as inappropriate with regard the new management objectives. The evolutionary change has a global impact.

Formalism defines the kind of the modeling paradigm adopted to specify the process definition (see §2.2): activity oriented, product oriented, decision/ intention oriented and conversation oriented. We remind here that decision oriented formalisms address the essential question on the BPs, "why", and allow us to define alternative organizational solutions for a given business objective [14], [18], [38]. It permits also to develop user-oriented systems [43]. The user is considered « knowledgeable » on the BP which can not be entirely pre-defined using control flows among activities. He/she has the possibility to select an alternative path during the enactment of the process instance or to decide about the operations to perform to meet his/hers responsibilities in the BP.

Transition defines if it exists a transition model (bridge) allowing moving a process instance from an 'old' process definition to a 'target' process definition [8], [47], [57], [60]. It is only applicable when the flexibility is $a$ posteriori and the impact of the change is global. The transition is one of the major problems in dynamic process modification: what happens with the current instances which definition is modified? This is closely related to the property of migration techniques.

Versioning defines if the approach handles several versions of the same process definition. It is only applicable when the flexibility is a posteriori. The versioning offers a means to support the understanding on 
BP evolution and to anticipate the future transformations [11], [28], [47].

Evolution techniques define how the evolution of process definitions or instances is performed. It is only applicable when the flexibility is a posteriori.

Ad hoc: a non pre-defined action on instances or definition is created during the execution to modify the behavior of the BP [1], [8], [11], [14], [27], [28], [47]. The target process definition is not known. The ad hoc change should be tolerated only on the instance level [58].

Derivation: the target process definition is known. The problem is to move the current instances. This often requires a transition model [8], [28], [47], [62].

Inheritance: it exploits the inheritance concept well known in object oriented modeling and extends it to the definition of generic process models [57], [60].

Induction: it allows process mining based on the execution of process instances [23]. The purpose is to adapt the process definition to the previously executed instances using an inductive learning mechanism.

Reflexion: it proposes to design process definitions which have the ability to control and to modify their own behavior, automatically or by user intervention. This is realized based on the feed-backs on the instances [8], [15].

Rule based: it allows the evolution of the process instances based on the events arrived during their performance [26], [47]. Events are known and are associated to corrective activities.

Migration techniques define what happens with the current instances which process definition is modified. This property is only applicable when the flexibility is $a$ posteriori and the nature of the impact is global.

Cancellation: the execution of the impacted instances is cancelled and new instances are created according to the new process definition. This is the least recommended technique because of the loss of information and time [28].

With propagation: the modification of the process definition is propagated to the current instances. This is the most complex technique because it requires a transition model allowing the conformity of the current instances with the target process definition [11], [28].

Without propagation: current instances continue their execution according to the old process definition, the new instances are executed according to the new process definition (requires versioning). The propagation of the modification to the instances can be deferred (delayed migration), i.e. instances are executed according to the old model until they reach a safe state. This strategy is used in Milano [1] and Wasa2 [62].

Flexibility techniques are applied during BP modeling in order to implement non prescriptive BP definitions which could be refined during the execution. This property is only applicable when the flexibility is a priori.

Late binding: the various elements of the $\mathrm{BP}$ are considered as objects which behavior is defined during the execution. No modification is required on the process definition but a large choice is provided among several execution alternatives [14]. This includes the association of activities to the most adequate implementation during the execution [27], the selection of the resource better satisfying a given criteria or the selection of the most suitable actor for carrying out an activity according to certain capacities. In AFLOWS [7], it is possible to associate the same process definition to different behavior models according to the execution context.

Late modeling: some parts of the BP model are left open to innovation and creativity of its users, especially when the corresponding specifications can not be well identified in advance. Some activities can be declared as critical and mandatory while some others could be optional and omitted during execution. It is also possible to specify the activities and to leave to the user the freedom of selecting their precedence relationships during the execution of the process instance. In [27] several behaviors can be associated to the activities. The user selects the most appropriate one in a dynamic way. In [28], the user selects the appropriate performance for an activity which was initially specified as a goal to achieve. If no existing method is appropriate, it is also possible to dynamically create a new one.

The case handling: this paradigm was proposed in [59] to support the flexible BPs mainly based on knowledge sharing (for knowledge intensive BPs). It offers a good balance between the data-centered approaches of the Eighties and the process-centered ones of the Nineties. Data and process are not separated as in traditional WFMSs. The principal characteristics and differences with the traditional WFMS lie in: (i) The case is considered by the participants as a whole and not like a sum of small parts. Knowledge sharing is thus essential. (ii) The activities to be performed are decided on the basis of available information, rather than on the activities already carried out. The case handling is data-driven instead of being exclusively control flow driven; the distribution of the tasks is done by a flexible mechanism, which makes it possible to the users to navigate through all activities to complete cases. (iii) Each activity is associated to three types of roles: the one who is in charge of its execution, the one who can undo it or the one who can skip it. We could argue that according to this paradigm, the build-time and the run-time of the process enactment life cycle as "opposed" in Figure 1 are in reality intertwined.

The case handling is not the only paradigm recommending a state driven view of workflows. In [25], an approach to process flexible workflows based on a state driven view is proposed. This view focuses on changes that each activity introduces in order to bring thus the process closer of its desired final state (its goal). A process is considered as a trajectory in the space of all possible states (flow of states). The occurrence of an external event 


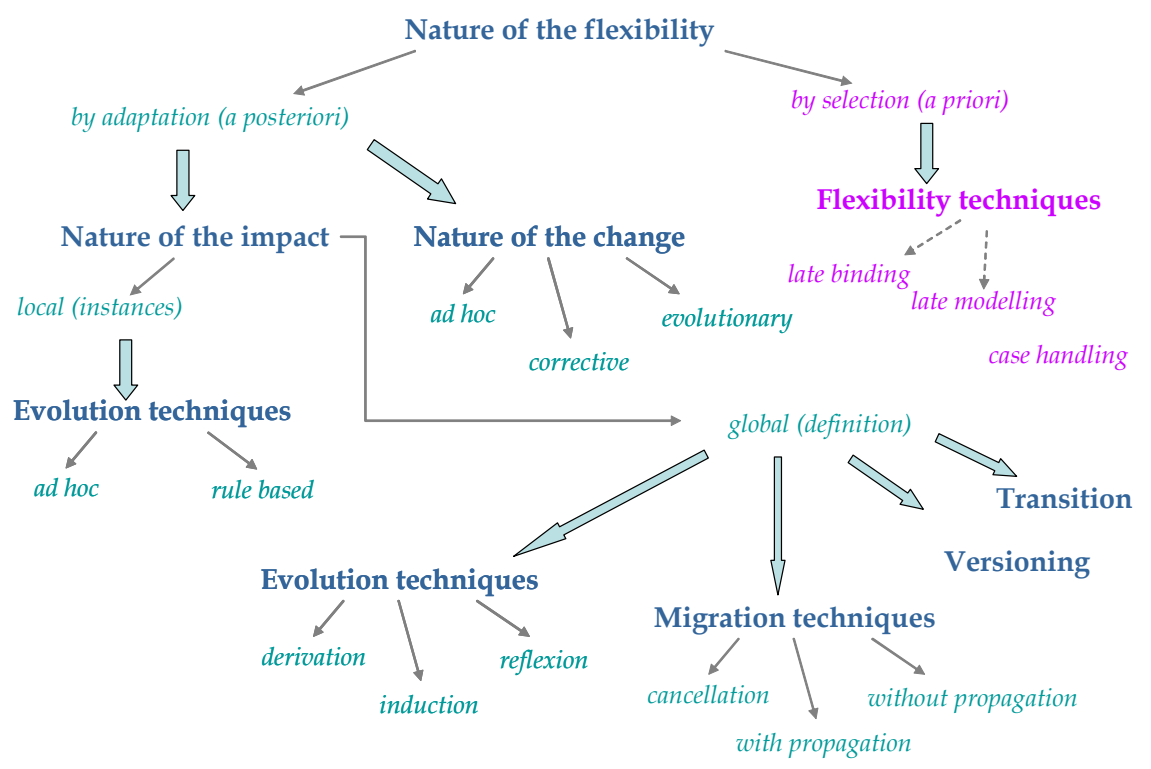

Figure 2: Summarizing flexibility requirements to be handled with the BP modeling and execution support systems

is a trigger to move the process from a state to another, towards the final state: the flow of activities that should be executed depends on how the current state differs from the projected final one. A set of planning rules classified into three categories (obligations, recommendations and prohibitions) regulates the task distribution and control.

\section{Conclusion and future work}

The notion of Enterprise modeling refers to a collection of conceptual modeling techniques for describing different facets of the organizational domain including operational, organizational and teleological considerations. Using models to represent the enterprise allows a coherent and complete description. These models are useful because they allow (i) to improve the knowledge about the enterprise, (ii) to reason on alternative solutions and diverging points of view, and (iii) to reach an agreement.

Our experience of BP Modeling, BP Reengineering and the design of the supporting ISs stands in a number of industrial projects and European research projects. The common points we found in all these projects were (i) the large amount of detail to be handled in analyzing and improving BPs and the difficulties for mastering it; and (ii) the prime importance of establishing and preserving the 'best fit' between organization needs (whys) and system functionalities (whats), i.e. between process models and IS specifications. In order to keep the alignment of BPs with the organisations' IS and strategies, BPs should embody a great amount of flexibility and variability. So that, BPs are better supported by the IS and implement well the strategic and organisational goals of the organization.
Even if process modeling was fully helpful for managers to improve operational performance, it also demonstrated to be insufficient to help organizations to deal with the challenge of competitiveness in a constantly changing environment. The intention driven process modeling provides basis for understanding and supporting the enterprise objectives, the alternative way-of-workings, and when required, the reasons of change. In fact, this should be completed with the realization conditions of these objectives, i.e. taking in consideration the organizational and operational choices in order to specify the requirements on the IT systems needed by this enterprise.

Similarly, a great amount of flexibility is brought by the concepts of role and context. Changes in pieces of works of several granularities can be done at the BP type and instance level. The subject of change can be associated with organizational, functional, behavioral and operational perspectives. Context sensitive BP models fit better the customers' expectations which are often context-dependant.

The requirements related to the nature of the flexibility, the nature of the impact and the nature of the change capture (i) the ability of the business processes (and of the organization) to change and (i) the capacity of the modeling formalisms to incorporate this ability into process representations. The requirements related to the evolution and migration techniques, the versioning and transition capture the ability of -and the means used bythe support systems to enact those process representations.

Our future work concerns the usage perspectives of the BP modeling and of the support systems in order to extend the taxonomy of flexibility requirements summarized on Figure 2. We are investigating on the following issues: 
- Do the various kinds of business processes (operational, control, strategic, support) require the same kind of flexibility? Should we provide the same methodological and technological means to achieve it?

- Do the dimensions of change (for instance dynamism, adaptability and flexibility according to [48]) depend on this organizational categorization of the business processes?

- What are the goals of the stakeholders which push them to develop business process management systems able to change? Do they wish to innovate, to improve, to personalize, to obtain guidance...

- Which perspectives of the organization require change: a domain, a process, an activity, responsibilities, an application component, etc...

- How the modeling artifacts should be adapted to deal with the flexibility requirements related to those issues?

\section{References}

[1] Agostini, A., De Michelis, G. A Light Workflow Management System Using Simple Process Models (2000) In Computer Supported Cooperative Work: The Journal of Collaborative Computing, Kluwer Academic Publishers, Vol.9, No.3-4, p. 335-363.

[2] Alonso, G., Agrawal, D., El Abbadi, A. and Mohan, C. (1997) Functionality and Limitations of Current Workflow Management Systems, IEEE Expert, 12(5).

[3] Balabko, P., Wegmann, A., Ruppen, A. and Clément, N. (2004) The Value of Roles in Modelling Business Processes. BPMDS'04. Riga, Latvia.

[4] Bandinelli, S., Fugetta, A. and Grigoli, S. (1993) Process Modelling in the large with SLANG. 2nd Int. Conference on Software Process, Berlin, Germany, p. 75-93.

[5] Barrios, J., Nurcan, S. (2004) Model Driven Architectures for Enterprise Information Systems, CAISE'04, Latvia, Springer Verlag (pub).

[6] Bassil, S., Keller, R.K., Kropf, P.G. (2004) A workfloworiented system architecture for the management of container transportation. In Desel, J., Pernici, B., Weske, M.,eds.: Business Process Management. Volume 3080 Lecture Notes in Computer Science., Springer, p. 116-131

[7] Belhajjame, K., Vargas-Solar, G. and Collet, C. (2001) Towards an Adaptable workflow management system. 17èmes Journées BDA, Agadir, Maroc.

[8] Borghoff, U.M., et al. (1997) Reflective Agents for Adaptive Workflows, 2d Int. Conference and Exhibition on Practical Application of Intelligent Agents and MultiAgents (PAAM'97) London, UK, Society Press, p. 405-420.

[9] Casati, F., Ceri, S., Pernici, B. and Pozzi, G. (1996) Workflow Evolution. 15th ER'96 international Conference, Oct 7-10, Cottbus, Germany, Springer Verlag.

[10] Charoy, F., Guabtni, A. and Valdes Faura, M. (2006) A Dynamic Workflow Management System for Coordination of Cooperative Activities, Business Process Management Workshops 2006, p. 205-216.

[11] Dadam, P., Reichert, M. and Rinderle, S. (2003) Evaluation of Correctness Criteria For Dynamic Workflow Changes. Int. Conference on Business Process Management (BPM'03), Eindhoven, The Netherlands, June 2003, p. 41-57.
[12] Daoudi, F. and Nurcan S. (2007) A benchmarking framework for methods to design flexible business processes. Special issue of the Software Process: Improvement and Practice Journal on "Business Process Management", January 2007.

[13] Decker, S., Daniel, M., Erdmann, M. and Studer, R. (1997) An enterprise reference scheme for integrating Model based knowledge engineering and enterprise modeling., EKAW’97, Springer-Verlag, Heidelberg.

[14] Dellen, B., Maurer, F., and Pews, G. (1997) Knowledge Based Techniques to Increase the Flexibility of Workflow Management. Data and Knowledge Engineering, 23(3) North Holland.

[15] Edmond, D. and ter Hofstede, A.H.M (1998). Achieving workflow adaptability by means of reflection. CSCW Towards Adaptive Workflow Systems Workshop, Seattle, WA, November 1998.

[16] Ellis C. (1979) Information Control Nets, A Mathematical Model of Office Information Flow, ACM conference on Simulation, Measurement and Modelling of Computer Systems, 1979, p.225-240.

[17] Espejo, R. and Harnden R. (eds) (1989). The Viable System Model: Interpretations and Applications of Stafford Beer's VSM, Chichester: Wiley.

[18] Faustmann, G. (1998) Enforcement vs. Freedom of ActionAn Integrated Approach to Flexible Workflow Enactment. Workshop on Adaptive Workflow Systems. Conference on CSCW, Seattle, USA.

[19] Gligor, V., Gavilla, S., and Ferraiolo, D. (1998) On the Formal Definition of Separation-of-Duty Policies and their Composition. IEEE Symposium on Security and Privacy.

[20] Gotel, O. and Finkelstein, A. USA (1996) An Analysis of the Requirements Traceability Problem. First IEEE International Conference ICRE'94, Colorado Springs.

[21] Grigori, D., Charoy, F. and Godart, C. (2004) COO-flow: a Process Technology to Support Cooperative Processes, Int. Jour. of Software Engineering and Knowledge Engineering, Special issue: Best Papers from SEKE 2003, 14(1).

[22] Harel, D. (1990) STATEMATE: A working environment for the development of complex reactive systems. IEEE Transactions on Software Engineering, 16:4, p.403-414.

[23] Herbst, J. and Karagiannis, D. (1999) An Inductive Approach to the Acquisition and Adaptation of Workflow Models. IJCAI'99 Workshop on Intelligent Workflow and Process Management: The New Frontier for AI in Business, p.52-57.

[24] Jarzabek, S. and Ling, T.W. (1996) Model-based support for business reengineering, Information and Software Technology, $\mathrm{N}^{\circ} 38,355-374$.

[25] Khomyakov, M. and Bider, I. (2001) Achieving workflow flexibility through taming the chaos, Journal of Conceptual Modeling, August 2001.

[26] Klein, M. and C. Dellarocas (2000) A Knowledge-Based Approach to Handling Exceptions in Workflow Systems. Journal of Computer-Supported Collaborative Work. Special Issue on Adaptive Workflow System, 9(3/4).

[27] Klingemann, J. (2000) Controlled flexibility in workflow management. 12th Int. Conf. on Advanced Information Systems Engineering (CAISE), Stockholm, Sweden, 126-141.

[28] Kradolfer, M. and A. Geppert (1999) Dynamic workflow schema evolution based on workflow type versioning and 
workflow migration, Int. Conf. on Cooperative Information Systems, Edinburgh. IEEE Computer Society Press, 104-114.

[29] Lehman, M.M. (1987) Process Models, Process Programs, Programming Support, 9th International Conference on Software Engineering.

[30] McCready, S. (1992) There is more than one kind of workflow software. Computerworld, November 1992.

[31] Mangan, P. and Sadiq, S. (2003) A constraint Specification Approach to Building flexible workflows. Journal of Research and Practice in Information Technology, 35:1.

[32] Marca, D.A. and McGowan, C.L. (1993) IDEF0/SADT: Business Process and Enterprise Modeling. San Diego: Eclectic Solutions, Inc.

[33] McLean, A., Moran, T. and Young, R.M. (1989) Design Rationale: The argument behind the artifact. CHI'93 Conference Proceedings, ACM, 247-256.

[34] Medina-Mora, R., Winograd, T., Flores, R., Flores, F. (1992) The Action Workflow Approach to Workflow Management Technology, CSCW'92, Toronto, Canada.

[35] Mendling J, Nüttgens M (2003) EPC Syntax Validation with XML Schema Languages. $2^{\text {nd }}$ GI-Workshop on Business Process Management with Event-Driven Process Chains (EPK 2003), Bamberg, Germany.

[36] Neiger, D. and Churilov, L. (2004) "Goal-Oriented Business Process Modeling with EPCs and Value-Focused Thinking”, "Lecture Notes in Computer Science”, Vol. 3080, 98-115.

[37] Nurcan, S. and Edme, M.-H. (2005) Intention Driven Modelling for Flexible Workflow Applications. Special issue of the Software Process: Improvement and Practice Journal on "Business Process Management, Development and Support", 10:4.

[38] Nurcan, S., Etien, A., Kaabi, A., Zoukar, I. and Rolland, C. (2005) A Strategy Driven Business Process Modelling Approach. Special issue of the Business Process Management Journal on "Goal-oriented business process modeling", Emerald, 11:6.

[39] Nurcan, S. (2004) Business Process Modeling for developing Process Oriented IT Systems, The "Business Process Management Tools and Technologies" track of the Int. IRMA Conf. May 23-26, 2004, New Orleans, USA.

[40] Nurcan, S. (1996) A method for cooperative information systems analysis and design: CISAD, Second International Conference on the Design of Cooperative Systems (COOP'96), 12-14 juin 1996, Juan-Les-Pins, p. 681-700.

[41] Ould, M.A. (1995) Business Processes: Modelling and analysis for re-engineering and improvement, John Wiley \& Sons.

[42] Regev, G., Wegmann, A. (2005) A Regulation-Based View on Business Process and Supporting System Flexibility, Proc. of the CAiSE'05 Workshop, p. 91-98.

[43] Robinson, W.N. (1996) Goal-Oriented Workflow Analysis and Infrastructure, NSF Workshop on Workflow \& Process Automation, Athens, GA.

[44] Rosemann, M., Recker, J. (2006) Context-aware Process Design Exploring the Extrinsic Drivers for Process Flexibility, BPMDS, Luxembourg.

[45] Ross, T.R. (1985) Applications and extensions of SADT, IEEE Computer, p. 25-34, April 1985.

[46] Rumbaugh, J., Blaha, M., Premerlani, W., Eddy, F. and Lorensen, W. (1991) Object Oriented Modeling and Design, Prentice-Hall.

[47] Sadiq, S.W and Orlowska, M.E. (1999) Architectural
Considerations in Systems Supporting Dynamic Workflow Modification. Workshop on Software Architectures for Business Process Management at CAiSE 1999, Heidelberg, Germany. June 14-18.

[48] Sadiq, S.W. (2002) Dynamically Changing Workflow Processes, $\mathrm{PhD}$ Thesis.

[49] Sadiq, S.W., Orlowska, M.E., Sadiq, S.W. (2005) Specification and validation of process constraints for flexible workflows. Inf. Syst. 30(5) 349-378.

[50] Saidani, O., Nurcan, S. (2006) A Role-Based Approach for Modelling Flexible Business Processes, The 7th Workshop on Business Process Modelling, Development, and Support (BPMDS'06), (in association with CAISE'06), Springer Verlag (pub), Luxembourg (2006).

[51] Saidani, O., Nurcan, S. (2007) Towards Context Aware Business Process Modelling. The 8th Workshop on Business Process Modelling, Development, and Support (BPMDS'07, (in association with CAISE'07), Springer Verlag (pub), June 11-12, 2007, Trondheim, Norway.

[52] Schmidt, R. (2005) Flexible Support of InterOrganizational Business Processes Using Web Services. Proceedings of the CAiSE'05 Workshop, p. 51-58.

[53] Searle, J.R. (1975) A taxonomy of illocutionary acts, K. Gunderson (Ed.), Language, mind and knowledge, Minneapolis, University of Minnesota Press, 334-369.

[54] Shi, D. and Danies, R.L. (2003) A survey of Manufacturing Flexibility: Implications For E-Business Flexibility”, IBM Systems Journal 42(3), p.414-427.

[55] Singh, B. and Rein., G. (1992) Role Interaction Nets (RINs): A process Description Formalism, MCC.

[56] Soffer, P. (2005) On the Notion of Flexibility in Business Processes. Proc. of the CAiSE'05 Workshop, p. 35- 42.

[57] Van der Aalst, W.M.P. (2001) How to Handle Dynamic Change and Capture Management Information: An Approach Based on Generic Workflow Models. Int. Journal of Computer Systems Science Engineering, 15(5).

[58] Van der Aalst W.M.P., Weske, M. and Wirtz, G. (2003) Advanced Topics in Workflow Management: Issues, Requirements, and Solutions. Journal of Integrated Design and Process Science, 7(3).

[59] Van der Aalst W.M.P., Stoffele, M. and Wamelink, J.W.F. (2003) Case Handling in Construction, Automation in Construction, 12(3), 303-320.

[60] Van der Aalst, W.M.P., Basten, T., Verbeek, H., Verkoulen, P. and Voorhoeve M. (1999) Adaptive Workflow: On the Interplay between Flexibility and Support. In J. Filipe and J. Cordeiro (editors), First Int. Conference on Enterprise Information Systems, 353-360, Setubal, Portugal.

[61] Weber, Rinderle, Reichert (2007) Change Patterns and Change Support Features in Process-Aware Information Systems, CAISE'07, Trondheim, Norway.

[62] Weske, M. (2001) Formal Foundation and Conceptual Design of Dynamic Adaptations in a Workflow Management System. 34th Annual Hawaii Int. Conf. on System Sciences (HICSS-34)-Volume 7, January 3-6..

[63] WfMC-TC-1011 v3 (1999) Workflow Terminology \& Glossary, February 1999.

[64] Yu, E.S.K. and Mylopoulos, J. (1994) From E-R to "A-R" Modelling Strategic Actor Relationships for Business Process Reengineering, 13th Int. Conference on the EntityRelationship Approach, December 13-16, Manchester. 